Research Article

\title{
High Lipoprotein(a) Level Is Independently Associated with Adverse Clinicopathological Features in Patients with Prostate Cancer
}

\author{
Fang-Ming Wang $\mathbb{D}^{1}$ and Yan Zhang $\mathbb{D}^{2}$ \\ ${ }^{1}$ Department of Urology, The Affiliated Hospital of Qingdao University, Qingdao, Shandong 266003, China \\ ${ }^{2}$ Division of Dyslipidemia, State Key Laboratory of Cardiovascular Disease, Fu Wai Hospital, National Center for \\ Cardiovascular Diseases, Chinese Academy of Medical Sciences, Peking Union Medical College, Beilishi Road 167, \\ Beijing 100037, China
}

Correspondence should be addressed to Fang-Ming Wang; qingyifangming@163.com and Yan Zhang; 18910162571@163.com

Fang-Ming Wang and Yan Zhang contributed equally to this work.

Received 16 July 2019; Revised 22 October 2019; Accepted 11 November 2019; Published 22 November 2019

Academic Editor: Timo Sorsa

Copyright (c) 2019 Fang-Ming Wang and Yan Zhang. This is an open access article distributed under the Creative Commons Attribution License, which permits unrestricted use, distribution, and reproduction in any medium, provided the original work is properly cited.

\begin{abstract}
Background. The effect of lipoprotein(a) (Lp(a)) on prostate cancer (PCa) is unclear. The aim of this study was to investigate the association between serum Lp(a) levels and clinicopathological features in patients with PCa. Methods. A total of 376 consecutive pathologically diagnosed PCa patients were enrolled and were classified as a low-intermediate-risk group or a high-risk group. The association of $\mathrm{Lp}(\mathrm{a})$ and the other lipid parameters including total cholesterol (TC), low-density lipoprotein cholesterol (LDL-C), high-density lipoprotein cholesterol (HDL-C), triglycerides (TG), TC/HDL-C, LDL-C/HDL-C, and remnant cholesterol (RC) with clinicopathological parameters was tested by univariate and multivariate logistic regression analyses. Results. The highrisk PCa patients tended to have higher Lp(a) levels $(p=0.022)$ while there was no significant difference regarding the other lipid parameters $(p>0.05)$ compared to low-intermediate-risk counterparts. Patients with PSA $\geq 100 \mathrm{ng} / \mathrm{ml}$ had significantly higher $\mathrm{Lp}(\mathrm{a})$ levels than subjects with PSA $<100 \mathrm{ng} / \mathrm{ml}(p=0.002)$. Univariate logistic regression analyses revealed that high $\mathrm{Lp}(\mathrm{a})$ levels were correlated with high-risk PCa (Q4 vs. Q1, HR $=2.687,95 \%$ CI: 1.113-6.491, $p=0.028$ ), while the other lipid parameters were not correlated with high-risk PCa. In the stepwise multivariate regression analysis, the association between Lp(a) levels and highrisk PCa remained significant (Q4 vs. Q1, HR $=2.890,95 \% \mathrm{CI}: 1.148-7.274, p=0.024$ ) after adjusting for confounding factors including age, body mass index, hypertension, diabetes, coronary artery disease, and lipid-lowering drugs. Conclusions. This is the first study showing the positive association between high Lp(a) and adverse clinicopathological features of PCa. PCa patients with high $\operatorname{Lp}(\mathrm{a})$ tends to be more aggressive and should receive more attention in clinical practice.
\end{abstract}

\section{Introduction}

Lipoprotein(a) $(\operatorname{Lp}(a))$ is a lipoprotein synthesized by the liver, consisting of a low-density lipoprotein (LDL) core with an apolipoprotein B-100 molecule covalently linked to apolipoprotein(a) (apo(a)) [1]. Lp(a) levels are essentially genetically determined and rather stable over time; they vary widely between individuals and show right-skewed distribu- tion in the population. Besides, $L p(a)$ levels are less influenced by diet or lipid-lowering drugs [2] but are closely related to female sex hormones and increased after the menopause [3]. Large-scale prospective cohort studies have shown that high plasma concentration of $\mathrm{Lp}(\mathrm{a})$ is a risk factor for cardiovascular diseases (CVD) and stroke [4-7]. Recently, increased attention has been paid to the effect of Lp(a) on tumors. Till now, some experimental studies 
support the antineoplastic effect of apo(a) or $\operatorname{Lp}(\mathrm{a})[8,9]$, but some clinical studies have reported contradictory results, demonstrating that higher cancer risk was observed for the highest $\mathrm{Lp}(\mathrm{a})$ levels in lung and colorectal cancers $[10,11]$.

To date, few studies have investigated potential associations between $\mathrm{Lp}(\mathrm{a})$ and the risk of prostate cancer (PCa). A recent study revealed that higher levels of $L p(a)$ were associated with an increase in PCa incidence risk [12]. Another study demonstrated that the lowest risk of PCa was observed for the highest levels of $\operatorname{Lp}(\mathrm{a})$ [11].

However, to the best of our knowledge, whether Lp(a) is associated with the clinicopathological characteristics of PCa has not been reported. Accordingly, in the present study, we sought to determine the association between $\operatorname{Lp}(\mathrm{a})$ and clinicopathological features including the risk severity of PCa in 376 pathologically diagnosed patients.

\section{Material and Methods}

2.1. Study Design and Population. We followed our previous methods [13]. The study was performed in accordance with the Declaration of Helsinki and was approved by the ethical committee of the Affiliated Hospital of Qingdao University. All subject names, initials, or hospital numbers were not used in the text, table, or illustrative materials of this study.

The study was conducted in patients with primary diagnosed, pathologically confirmed sporadic PCa, between January 2011 and October 2018 at the Department of Urology at the Affiliated Hospital of Qingdao University. All patients were Chinese Han people. The exclusion criteria of the study were the presence of medical history of other malignancies, severe liver and/or renal insufficiency, or incomplete clinicopathological information. All data on age, body mass index (BMI), history of hypertension, diabetes, coronary artery disease (CAD), lipid-lowering drugs, serum PSA, cancer grade, tumor clinical stage at diagnosis, treatment protocols, and lipid profiles were obtained from electronic records and medical charts. All the pathological data analyzed in this study were identified from the transrectal ultrasound-guided prostate biopsy or radical prostatectomy specimens. All specimens were processed according to standard pathological procedures. The tumor stage was assessed according to the American Joint Committee on Cancer (AJCC) TNM classification of malignant tumors 2002. Cancer grade was assessed according to the ISUP classification of 2014 [14].

2.2. Blood Sampling and Lipoprotein(a) Measurement. Venous blood samples were collected from all subjects after overnight fasting according to a standardized protocol. Blood samples were obtained from PCa patients before surgical management, androgen deprivation, or other therapies. $\mathrm{Lp}$ (a) levels were measured by the turbidimetric immunoassay method (LASAY Lp(a) auto; SHIMA Laboratories Co., $\mathrm{Ltd}$.) with a normal value of $<30 \mathrm{mg} / \mathrm{dl}$. An $\mathrm{Lp}$ (a) protein validated standard was used to calibrate the examination, and the coefficient of variation value of repetitive measurements was $<10 \%$. Total cholesterol (TC), high-density lipoprotein cholesterol (HDL-C), low-density lipoprotein cholesterol
(LDL-C), and triglycerides (TG) were determined using enzymatic methods on an Hitachi 7600D clinical chemistry analyzer according to the manufacturer's instructions. Remnant cholesterol (RC) was calculated by TC - HDL - LDL.

2.3. Statistical Analyses. Quantitative variables were expressed as mean \pm standard deviation (SD) except for $\mathrm{Lp}(\mathrm{a})$ and TG, which were expressed as median with interquartile range, and were analyzed by Student's $t$-tests, oneway ANOVA, Mann-Whitney $U$ tests, or Kruskal-Wallis tests as appropriate. The qualitative variables were expressed as numbers and percentages and were analyzed by chisquared statistic tests. All PCa subjects were divided into two groups: low-intermediate-risk group and high-risk group according to the PSA, Gleason score, and clinical stage. Lowintermediate risk is defined as follows: PSA $\leq 20 \mathrm{ng} / \mathrm{ml}$, ISUP grade $\leq 3$, and clinical stage $\leq \mathrm{T} 2 \mathrm{~b}$; high risk was based on either one of the following criteria: PSA $>20 \mathrm{ng} / \mathrm{ml}$, ISUP grade $\geq 4$, or clinical stage $\geq \mathrm{T} 2 \mathrm{c}$. The stratification of PCa risk was conducted by two surgeons, and they were blind to $\mathrm{Lp}(\mathrm{a})$ levels for these same patients. Correlations between variables with high-risk PCa were examined by univariate logistic regression analysis. Stepwise multivariate regression analysis was used to determine the independent factors of high-risk PCa with adjustment for potential confounding factors including age, BMI, hypertension, diabetes, CAD, and lipid-lowering drugs.

Besides, we divided $\mathrm{Lp}(\mathrm{a})$ into four quartiles as follows: the first quartile group (Q1, <104 mg/l (25th percentile); $n=95$ ), the second quartile group (Q2, 104-216 mg/l (25-50th percentile); $n=93$ ), the third quartile group (Q3, 216-381 mg/l (50-75th percentile); $n=94)$, and the fourth quartile group (Q4, >381 mg/l (75-100th percentile); $n=94)$, and analyzed clinicopathological features in different levels of $\mathrm{Lp}(\mathrm{a})$. A $p$ value of less than 0.05 was considered statistically significant. Statistical studies were carried out with the SPSS program (version 19.0, SPSS, Chicago, Illinois, USA).

\section{Results}

3.1. Baseline Characteristics. The current study consisted of 376 eligible pathologically confirmed PCa patients (male, with a mean age of $70.8 \pm 7.9$ years), including 54 cases of low-intermediate-risk and 322 cases of high-risk subjects.

The baseline demographic, clinical characteristics, and laboratory findings of the enrolled subjects are summarized in Table 1. In brief, no significant differences in age, BMI, hypertension, diabetes, CAD, and lipid-lowering drugs were observed between low-intermediate- and high-risk groups.

$\mathrm{Lp}$ (a) levels of the high-risk group were significantly higher than those of the low-intermediate-risk group (224 (110-401) versus 165 (71-334), $p=0.022)$. However, there was no significant difference regarding TG, TC, LDL-C, HDL-C, TC/HDL-C, LDL-C/HDL-C, or RC between lowintermediate- and high-risk groups $(p>0.05)$.

To evaluate the lipid characteristics according to PSA levels, we classified all subjects into two groups: PSA $<100$ $\mathrm{ng} / \mathrm{ml}(n=220)$ and PSA $\geq 100 \mathrm{ng} / \mathrm{ml}(n=156)$. As shown 
TABLE 1: Baseline characteristics of the study subjects according to the risk of PCa.

\begin{tabular}{|c|c|c|c|c|}
\hline & All subjects $(n=376)$ & Low-intermediate risk $(n=54)$ & High risk $(n=322)$ & $p$ value \\
\hline \multicolumn{5}{|l|}{ Demographic characteristics } \\
\hline Age (years) & $70.8 \pm 7.9$ & $69.8 \pm 5.8$ & $70.9 \pm 8.2$ & 0.230 \\
\hline BMI $\left(\mathrm{kg} / \mathrm{m}^{2}\right)$ & $24.5 \pm 3.4$ & $24.7 \pm 3.4$ & $24.4 \pm 3.4$ & 0.639 \\
\hline Hypertension (n (\%)) & $140(37.2)$ & $19(35.2)$ & $121(37.6)$ & 0.764 \\
\hline Diabetes mellitus ( $n(\%))$ & $49(13.0)$ & $10(18.5)$ & $39(12.1)$ & 0.273 \\
\hline Coronary artery disease $(n(\%))$ & $56(14.9)$ & $6(11.1)$ & $50(15.5)$ & 0.424 \\
\hline Lipid-lowering drugs $(n(\%))$ & $9(2.4)$ & $2(3.7)$ & $7(2.2)$ & 0.623 \\
\hline \multicolumn{5}{|l|}{ Lipid parameters } \\
\hline TG (mmol/l) & $1.04(0.76-1.39)$ & $1.07(0.68-1.49)$ & $1.04(0.77-1.38)$ & 0.520 \\
\hline TC $(\mathrm{mmol} / \mathrm{l})$ & $4.85 \pm 0.97$ & $4.91 \pm 0.91$ & $4.84 \pm 0.98$ & 0.634 \\
\hline LDL-C (mmol/l) & $2.79 \pm 0.75$ & $2.88 \pm 0.75$ & $2.78 \pm 0.75$ & 0.359 \\
\hline HDL-C (mmol/l) & $1.35 \pm 0.31$ & $1.34 \pm 0.34$ & $1.36 \pm 0.30$ & 0.714 \\
\hline TC/HDL-C & $3.69 \pm 0.88$ & $3.83 \pm 0.98$ & $3.67 \pm 0.87$ & 0.213 \\
\hline LDL-C/HDL-C & $2.15 \pm 0.69$ & $2.27 \pm 0.75$ & $2.12 \pm 0.68$ & 0.141 \\
\hline $\mathrm{RC}(\mathrm{mmol} / \mathrm{l})$ & $0.71 \pm 0.34$ & $0.70 \pm 0.33$ & $0.71 \pm 0.35$ & 0.903 \\
\hline Lp(a) (mg/l) & $216(104-382)$ & $165(71-334)$ & $224(110-401)$ & 0.022 \\
\hline \multicolumn{5}{|l|}{ Surgery $(n(\%))$} \\
\hline Radical prostatectomy & $75(19.9)$ & $26(48.1)$ & $49(15.2)$ & $<0.001$ \\
\hline Bilateral orchiectomy & $55(14.4)$ & 0 & $54(16.8)$ & 0.001 \\
\hline
\end{tabular}

Data are expressed as $n(\%)$, mean $\pm \mathrm{SD}$, or median (25th-75th percentile). The bold value indicated statistical significance. $\mathrm{PCa}=$ prostate cancer; $\mathrm{BMI}=$ body mass index; PSA $=$ prostate-specific antigen; $\mathrm{RC}=$ remnant cholesterol; $\mathrm{TC}=$ total cholesterol; HDL-C $=$ high-density lipoprotein cholesterol; LDL-C = low-density lipoprotein cholesterol; $T G=$ triglycerides; $L p(a)=$ lipoprotein(a).

TABLE 2: Lipid parameters of the study population stratified by PSA values.

\begin{tabular}{lccc}
\hline & & PSA values & \\
& PSA $<100(n=220)$ & PSA $\geq 100(n=156)$ & \\
\hline Demographic characteristics & & $70.0 \pm 8.2$ & 0.071 \\
Age (years) & $71.4 \pm 7.7$ & $23.8 \pm 3.1$ & $\mathbf{0 . 0 0 1}$ \\
BMI $\left(\mathrm{kg} / \mathrm{m}^{2}\right)$ & $25.0 \pm 3.5$ & $54(34.6)$ & 0.448 \\
Hypertension $(n(\%))$ & $85(38.6)$ & $18(11.5)$ & $17(10.9)$ \\
Diabetes mellitus $(n(\%))$ & $31(14.1)$ & $2(1.3)$ \\
Coronary artery disease $(n(\%))$ & $39(17.7)$ & $1.05(0.78-1.39)$ \\
Lipid-lowering drugs $(n(\%))$ & $7(3.2)$ & $4.77 \pm 1.00$ & 0.535 \\
Lipid parameters & & $2.72 \pm 0.76$ \\
TG (mmol/l) & $1.04(0.73-1.39)$ & $1.32 \pm 0.29$ \\
TC (mmol/l) & $4.90 \pm 0.95$ & $3.71 \pm 0.90$ \\
LDL-C (mmol/l) & $2.84 \pm 0.75$ & 0.315 \\
HDL-C (mmol/l) & $1.38 \pm 0.32$ & $2.14 \pm 0.71$ \\
TC/HDL-C & $3.68 \pm 0.88$ & $0.73 \pm 0.36$ \\
LDL-C/HDL-C & $2.15 \pm 0.68$ & 0.180 \\
RC (mmol/l) & $0.69 \pm 0.33$ & $253.0(139.3-445.8)$ \\
Lp(a) (mg/l) & $188(90-359)$ & 0.140 \\
\hline
\end{tabular}

Data are expressed as $n(\%)$, mean $\pm \mathrm{SD}$, or median (25th-75th percentile). The bold value indicated statistical significance. PCa=prostate cancer; $\mathrm{BMI}=$ body mass index; PSA = prostate-specific antigen; $\mathrm{RC}=$ remnant cholesterol; $\mathrm{TC}=$ total cholesterol; HDL-C $=$ high-density lipoprotein cholesterol; LDL-C = low-density lipoprotein cholesterol; TG = triglycerides; $\mathrm{Lp}(\mathrm{a})=$ lipoprotein(a) 
TABLE 3: Demographic and clinicopathological characteristics of study subjects according to the quartiles of Lp(a) plasma levels.

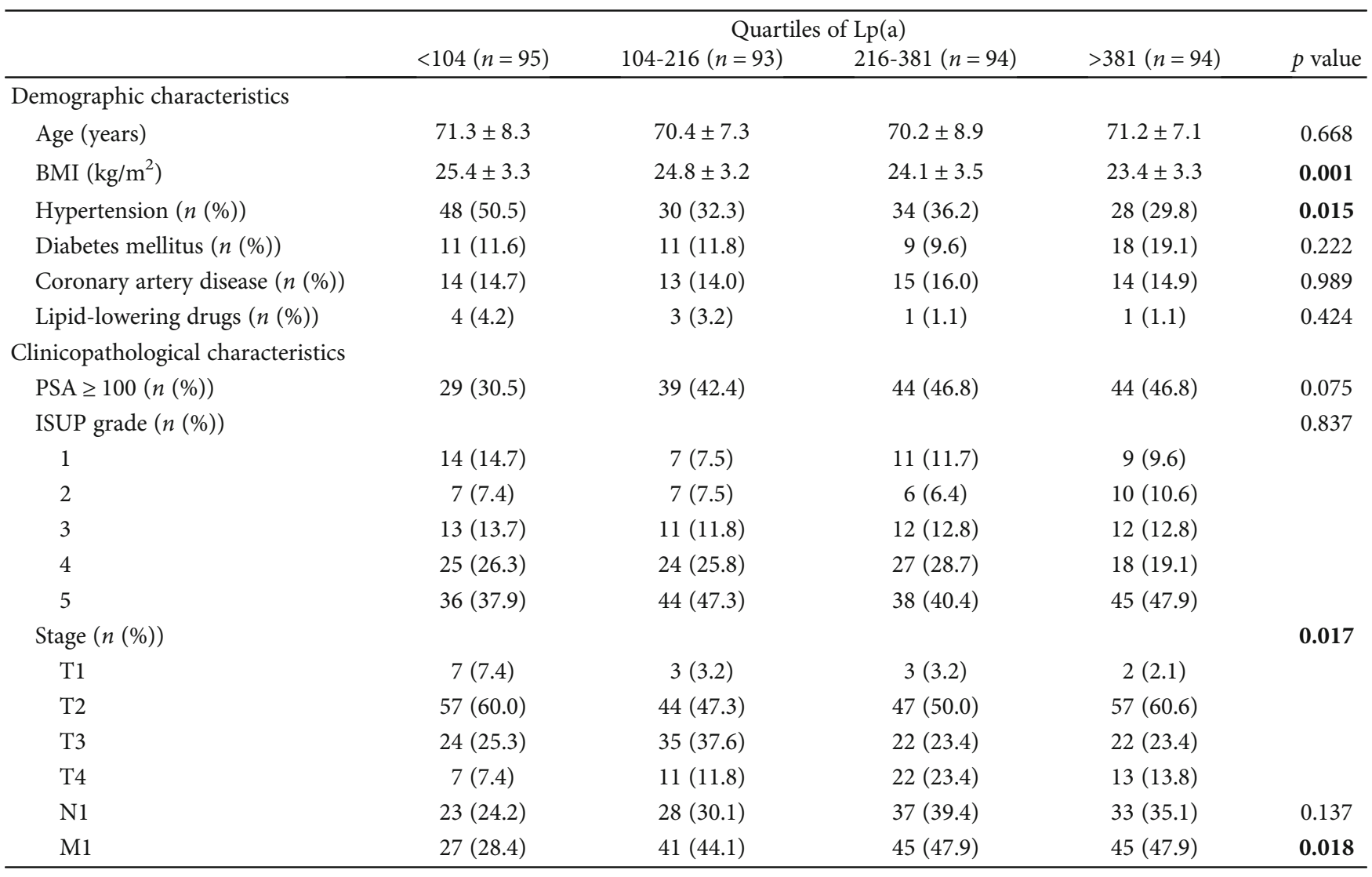

Data are expressed as $n$ (\%), mean \pm SD, or median (25th-75th percentile). The bold value indicated statistical significance. PCa $=$ prostate cancer; BMI $=$ body mass index; PSA = prostate-specific antigen; ISUP = International Society of Urological Pathology.

in Table 2, we observed that patients with PSA $\geq 100 \mathrm{ng} / \mathrm{ml}$ had a significantly higher Lp(a) level but a lower BMI compared to those with PSA $<100 \mathrm{ng} / \mathrm{ml}(253.0(139.3-445.8)$ $\mathrm{ng} / \mathrm{ml}$ versus 188 (90-359) $\mathrm{ng} / \mathrm{ml}, p=0.002 ; 23.8 \pm 3.1$ versus $25.0 \pm 3.5, p=0.001$, respectively). There was no significant difference regarding TG, TC, LDL-C, HDL-C, TC/HDL-C, LDL-C/HDL-C, or RC $(p=0.888, p=0.180, p=0.140$, $p=0.099, p=0.764, p=0.919$, and $p=0.279$, respectively).

3.2. Clinicopathological Characteristics in Different $L p(a)$ Levels. PCa patients were divided into four groups in terms of serum Lp(a) level quartiles as mentioned above. Statistically significant differences in BMI, hypertension, stage, and metastasis were found among different groups $(p=0.001$, $p=0.015, p=0.017$, and $p=0.018$, respectively). Specifically, the percentage of PCa metastasis increased gradually with the elevation of $\mathrm{Lp}(\mathrm{a})$ level and was highest in Q3 and Q4, a statistically significant difference compared with the other groups $(p=0.014)$, while BMI decreased with the elevation of $\operatorname{Lp}(\mathrm{a})$ level $(p=0.011)$. There is an evident trend that PSA $\geq 100 \mathrm{ng} / \mathrm{ml}$ and lymph node involvement percentage rose with the elevation of $\operatorname{Lp}(\mathrm{a})$ level although the difference was not statistically significant $(p=0.075, p=0.137)$. There were no differences in age, diabetes, CAD, or ISUP grade among the groups (Table 3 ).
3.3. Correlations of $\operatorname{Lp}(a)$ and the Other Lipid Parameters with High-Risk PCa. To evaluate the correlations of $\operatorname{Lp}(\mathrm{a})$ and other lipid parameters with high-risk $\mathrm{PCa}$, univariate logistic regression analysis was performed in the current study. As shown in Table 4, Lp(a) found to be statistically significant in univariate analyses was entered into multivariate logistic regression analysis. The data indicated that the high $\mathrm{Lp}$ (a) level was still independently correlated with the presence of high-risk PCa after adjusting for confounders including age, $\mathrm{BMI}$, hypertension, diabetes, $\mathrm{CAD}$, and lipidlowering drugs in multivariate logistic regression analysis (Q4 vs. Q1, OR =2.890, 95\% CI: $1.148-7.274, p=0.024$ ) (Table 4). Nevertheless, we did not observe a statistically significant relationship between high-risk $\mathrm{PCa}$ and other lipid parameters including TG, TC, LDL-C, HDL-C, TC/HDL-C, LDL-C/HDL-C, and RC (Table 4).

\section{Discussion}

The present study evaluated the association between high $\mathrm{Lp}(\mathrm{a})$ and adverse clinicopathological features of PCa. To our knowledge, this is the first study to demonstrate that patients with high $\mathrm{Lp}(\mathrm{a})$ tended to have higher risk of aggressive PCa compared with those with low Lp(a). This association remained significant after adjusting for other 
TABLE 4: Univariate and multivariate analyses to identify the independent correlation between high Lp(a) and high risk of PCa.

\begin{tabular}{lccc}
\hline & $\begin{array}{c}\text { Univariate model } \\
\text { OR }(95 \% \mathrm{CI})\end{array}$ & $\begin{array}{c}\text { Multivariate model } \\
\text { OR }(95 \% \text { CI })\end{array}$ \\
\hline TG $(\mathrm{mmol} / \mathrm{l})$ & $1.154(0.700-1.902)$ & 0.575 & \\
TC $(\mathrm{mmol} / \mathrm{l})$ & $0.931(0.694-1.249)$ & 0.633 & \\
LDL-C (mmol/l) & $0.839(0.578-1.220)$ & 0.358 & \\
HDL-C (mmol/l) & $1.196(0.460-3.110)$ & 0.714 & \\
TC/HDL-C & $0.818(0.596-1.122)$ & 0.213 & \\
LDL-C/HDL-C & $0.738(0.493-1.106)$ & 0.141 & 1 \\
RC (mmol/l) & $1.054(0.451-2.465)$ & 0.903 & \\
Lp(a) (mg/l) & & & $1.957(0.862-4.442)$ \\
Quartile 1 & 1 & 0.130 & $1.239(0.581-2.641)$ \\
Quartile 2 & $1.864(0.833-4.170)$ & 0.598 & $2.890(1.148-7.274)$ \\
Quartile 3 & $1.219(0.584-2.545)$ & $\mathbf{0 . 0 2 8}$ & 0.109 \\
Quartile 4 & $2.687(1.113-6.491)$ & 0.579 \\
\hline
\end{tabular}

Logistic regression analyses were performed. The multivariate model was adjusted for age, BMI, hypertension, diabetes, coronary artery disease, and lipid-lowering drugs. The bold value indicated statistical significance. $\mathrm{PCa}=$ prostate cancer; $\mathrm{RC}=$ remnant cholesterol; $\mathrm{TC}=$ total cholesterol; $\mathrm{HDL}-\mathrm{C}=$ highdensity lipoprotein cholesterol; LDL-C = low-density lipoprotein cholesterol; TG = triglycerides; Lp(a) = lipoprotein(a).

confounders. Therefore, the current study might have potential clinical implications with regard to the assessment of $\mathrm{PCa}$ risk based on the lipid perspectives. For example, for one $\mathrm{PCa}$ patient with high $\mathrm{Lp}(\mathrm{a})$, he should be followed up more closely and receive more attention in clinical practice. Besides, our study provided novel information to explore more possible mechanisms of PCa.

There is no doubt that circulating levels of $\operatorname{Lp}(\mathrm{a})$ and other lipid parameters are associated with CVD and are considered major indicators of metabolic health $[6,12,15]$. Recently, more and more research has focused on the relationship between $\mathrm{Lp}(\mathrm{a})$ and multiple kinds of tumors. Results from different studies are not entirely consistent. It is reported that patients with lung or breast cancer exhibit elevated $L p(a)$ levels $[10,16]$, while the $L p(a)$ level was relatively low in patients with hepatocellular carcinoma [17]. Marrer et al. [11] conducted a cohort study analyzing cancer incidence in relation to $L p(a)$ plasma levels and found that men with the highest $\mathrm{Lp}(\mathrm{a})$ levels seemed to have the highest risk of all-site, lung, colorectal, or tobacco/alcohol-related cancers, but the lowest risk of PCa. One could expect that if $\mathrm{Lp}(\mathrm{a})$ levels increase the incidence of cancer, they would also increase cancer mortality. However, a Japanese cohort study analyzing the association between $\operatorname{Lp}(\mathrm{a})$ levels and cancer deaths found an increased risk for low $L p(a)$ levels, when comparing the lowest quartile versus the three upper quartiles combined [18].

However, few studies on the relationship between $\mathrm{Lp}(\mathrm{a})$ and PCa have been reported. We reviewed the literatures and found that Katzke and colleagues [12] had reported that high $\mathrm{Lp}(\mathrm{a})$ levels were significantly associated with a 1.5 higher risk of incident $\mathrm{PCa}(\mathrm{Q} 4$ vs. $\mathrm{Q} 1, \mathrm{HR}=1.47)$. On the contrary, as mentioned above, Marrer et al. [11] demonstrated that the lowest risk of PCa was observed for the highest levels of $\mathrm{Lp}(\mathrm{a})$. Most studies compared the PCa patients with normal people and did not reveal the relationship between $\mathrm{Lp}(\mathrm{a})$ and risk of aggressiveness or invasion within patients with PCa.
Our study divided PCa patients into two groups: lowintermediate-risk group and high-risk group, then analyzed lipid parameters in the two groups, and found that $L p(a)$ was the only lipid parameter that differs between the two groups. Next, we demonstrated, by both univariate and multivariate analyses, that high $\mathrm{Lp}(\mathrm{a})$ independently correlated with the presence of high-risk PCa. Besides, we found that BMI was significantly lower in the high-Lp(a) group and PSA $\geq 100 \mathrm{ng} / \mathrm{ml}$ group than in the low-Lp(a) group and $\mathrm{PSA}<100 \mathrm{ng} / \mathrm{ml}$ group, which could be explained by the excessive energy consumption and weight loss in advanced PCa. Although there were no significant differences on positive pelvic lymph node between individuals with different levels of $\operatorname{Lp}(\mathrm{a})$, we can see a trend that patients with high Lp(a) (Q3 and Q4) had higher risk of lymph node metastasis, which was consistent with the significant result of bone metastasis.

There lack studies focusing on the relationship of $\operatorname{Lp}(\mathrm{a})$ and clinicopathological features in cancers. Our findings are similar to one recent study [19] in liver cancer, which revealed that hepatocellular carcinoma patients with portal vein thrombosis showed a statistical significant serum $L p(a)$ level higher than those without portal vein thrombosis.

The potential mechanisms of this correlation of high $\mathrm{Lp}(\mathrm{a})$ and adverse clinicopathological features of PCa remain uncertain and need further studies. $L p(a)$ plasma levels are genetically determined, affected only to a minor extent by age, sex, and environmental factors, and rather stable in individuals $[2,20]$. We speculate that there are two main completely different mechanisms to explain our study results. Firstly, some studies on animal models have indicated that the proteolytic breakdown products of $L p(a)$ possess antitumoral properties both in vitro and in vivo [21-23]. Considering the antitumoral role of $\mathrm{Lp}(\mathrm{a})$, we believe that the elevated Lp(a) levels in high-risk and PSA $\geq 100$ PCa patients might be compensatory reactions to chronic inflammation of the whole body caused by aggressiveness and invasion of a tumor. Secondly, apo(a), as an important component of 
$\mathrm{Lp}(\mathrm{a})$, is essentially composed of structural homologues to kringle IV of the plasminogen molecule and functions as a competitive inhibitor for the activation of plasmin-induced fibrinolysis $[24,25]$. Therefore, high $\operatorname{Lp}(\mathrm{a})$ could more easily induce the formation of fibrin network and thrombus, facilitating cancer cell adhesion, invasion, and metastasis. Moreover, hormonal imbalance may affect $\operatorname{Lp}(\mathrm{a})$ levels in the context of prostate cancer.

There were several limitations in our study. Firstly, it was conducted at a single center. Secondly, this was an observational study and could not identify the causal relationship. Finally, we did not evaluate the prognostic value of $L p(a)$ in the present study.

\section{Conclusions}

In conclusion, this study provided evidence of an association between $\mathrm{Lp}(\mathrm{a})$ and clinicopathological features of PCa: patients with high $\mathrm{Lp}(\mathrm{a})$ had more adverse clinicopathological features of PCa than those with low Lp(a). This association remained significant after adjustment for other confounding factors. Further basic research on PCa genetic or biological differences associated with $\operatorname{Lp}(\mathrm{a})$ is needed in the future studies.

\section{Data Availability}

The raw data that was used in this study is available upon request from the corresponding authors.

\section{Conflicts of Interest}

The authors declare that they have no conflicts of interests.

\section{Authors' Contributions}

Fang-Ming Wang and Yan Zhang equally contributed to the study.

\section{Acknowledgments}

This work was supported by the Specialized Research Fund for the Doctoral Program of the Affiliated Hospital of Qingdao University (111520) awarded to Dr. Fang-Ming Wang, MD, PhD.

\section{References}

[1] K. M. Kostner and G. M. Kostner, "Lipoprotein(a): still an enigma?," Current Opinion in Lipidology, vol. 13, no. 4, pp. 391-396, 2002.

[2] C. Sandholzer, D. M. Hallman, N. Saha et al., "Effects of the apolipoprotein(a) size polymorphism on the lipoprotein(a) concentration in 7 ethnic groups," Human Genetics, vol. 86, no. 6, pp. 607-614, 1991.

[3] P. L. Torng, T. C. Su, F. C. Sung et al., "Effects of menopause and obesity on lipid profiles in middle-aged Taiwanese women: the Chin-Shan Community Cardiovascular Cohort Study," Atherosclerosis, vol. 153, no. 2, pp. 413-421, 2000.
[4] J. Danesh, R. Collins, and R. Peto, "Lipoprotein(a) and coronary heart disease. Meta-analysis of prospective studies," Circulation, vol. 102, no. 10, pp. 1082-1085, 2000.

[5] B. Smolders, R. Lemmens, and V. Thijs, "Lipoprotein (a) and Stroke," Stroke, vol. 38, no. 6, pp. 1959-1966, 2007.

[6] B. G. Nordestgaard, M. J. Chapman, K. Ray et al., "Lipoprotein(a) as a cardiovascular risk factor: current status," European Heart Journal, vol. 31, no. 23, pp. 2844-2853, 2010.

[7] M. Sawabe, N. Tanaka, K. Nakahara et al., "High lipoprotein(a) level promotes both coronary atherosclerosis and myocardial infarction: a path analysis using a large number of autopsy cases," Heart, vol. 95, no. 24, pp. 1997-2002, 2009.

[8] J. Cha, M. W. Roomi, T. Kalinovsky, A. Niedzwiecki, and M. Rath, "Lipoprotein(a) and vitamin C impair development of breast cancer tumors in $\mathrm{Lp}(\mathrm{a})+$; Gulo-/- mice," International Journal of Oncology, vol. 49, no. 3, pp. 895-902, 2016.

[9] G. Lippi, M. Franchini, G. L. Salvagno, and G. C. Guidi, "Lipoprotein[a] and cancer: anti-neoplastic effect besides its cardiovascular potency," Cancer Treatment Reviews, vol. 33, no. 5, pp. 427-436, 2007.

[10] H. H. Yang, X. F. Chen, W. Hu et al., "Lipoprotein(a) level and its association with tumor stage in male patients with primary lung cancer," Clinical Chemistry and Laboratory Medicine, vol. 47, no. 4, pp. 452-457, 2009.

[11] É. Marrer, A. Wagner, M. Montaye et al., "Lipoprotein(a) plasma levels and the risk of cancer: the PRIME study," European Journal of Cancer Prevention, vol. 22, no. 3, pp. 286-293, 2013.

[12] V. A. Katzke, D. Sookthai, T. Johnson, T. Kühn, and R. Kaaks, "Blood lipids and lipoproteins in relation to incidence and mortality risks for CVD and cancer in the prospective EPICHeidelberg cohort," BMC Medicine, vol. 15, no. 1, p. 218, 2017.

[13] F. M. Wang, Y. Zhang, G. M. Zhang, Y. N. Liu, L. J. Sun, and Y. Liu, "Association of ABO blood types and clinicopathological features of prostate cancer," Disease Markers, vol. 2017, Article ID 9237481, 6 pages, 2017, Epub 2017 Oct 9.

[14] J. I. Epstein, L. Egevad, M. B. Amin et al., "The 2014 International Society of Urological Pathology (ISUP) consensus conference on Gleason grading of prostatic carcinoma: definition of grading patterns and proposal for a new grading system," The American Journal of Surgical Pathology, vol. 40, no. 2, pp. 244-252, 2016.

[15] S. Li, N. Q. Wu, C. G. Zhu et al., "Significance of lipoprotein(a) levels in familial hypercholesterolemia and coronary artery disease," Atherosclerosis, vol. 260, pp. 67-74, 2017.

[16] E. Kökoğlu, I. Karaarslan, H. M. Karaarslan, and H. Baloğlu, "Elevated serum Lp(a) levels in the early and advanced stages of breast cancer," Cancer Biochemistry Biophysics, vol. 14, no. 2, pp. 133-136, 1994.

[17] M. Motta, I. Giugno, P. Ruello, G. Pistone, I. di Fazio, and M. Malaguarnera, "Lipoprotein (a) behaviour in patients with hepatocellular carcinoma," Minerva Medica, vol. 92, no. 5, pp. 301-305, 2001.

[18] M. Sawabe, N. Tanaka, M. N. Mieno et al., "Low lipoprotein(a) concentration is associated with cancer and all-cause deaths: a population-based cohort study (the JMS cohort study)," PLoS One, vol. 7, no. 4, article e31954, 2012.

[19] G. Malaguarnera, V. E. Catania, A. Francaviglia et al., "Lipoprotein(a) in patients with hepatocellular carcinoma and portal vein thrombosis," Aging Clinical and Experimental Research, vol. 29, Suppl 1, pp. 185-190, 2017. 
[20] L. Berglund and R. Ramakrishnan, "Lipoprotein(a): an elusive cardiovascular risk factor," Arteriosclerosis, Thrombosis, and Vascular Biology, vol. 24, no. 12, pp. 2219-2226, 2004.

[21] J. S. Kim, J. H. Chang, H. K. Yu et al., "Inhibition of angiogenesis and angiogenesis-dependent tumor growth by the cryptic kringle fragments of human apolipoprotein(a)," The Journal of Biological Chemistry, vol. 278, no. 31, pp. 29000-29008, 2003.

[22] H. K. Yu, J. H. Ahn, H. J. Lee et al., "Expression of human apolipoprotein(a) kringles in colon cancer cells suppresses angiogenesis-dependent tumor growth and peritoneal dissemination," The Journal of Gene Medicine, vol. 7, no. 1, pp. 39-49, 2005.

[23] K. Lee, S. T. Yun, Y. G. Kim, Y. Yoon, and E. C. Jo, “Adenoassociated virus-mediated expression of apolipoprotein (a) kringles suppresses hepatocellular carcinoma growth in mice," Hepatology, vol. 43, no. 5, pp. 1063-1073, 2006.

[24] M. L. Wahl, D. J. Kenan, M. Gonzalez-Gronow, and S. V. Pizzo, "Angiostatin's molecular mechanism: aspects of specificity and regulation elucidated," Journal of Cellular Biochemistry, vol. 96, no. 2, pp. 242-261, 2005.

[25] M. A. Hancock, M. B. Boffa, S. M. Marcovina, M. E. Nesheim, and M. L. Koschinsky, "Inhibition of plasminogen activation by lipoprotein(a): critical domains in apolipoprotein(a) and mechanism of inhibition on fibrin and degraded fibrin surfaces," The Journal of Biological Chemistry, vol. 278, no. 26, pp. 23260-23269, 2003. 


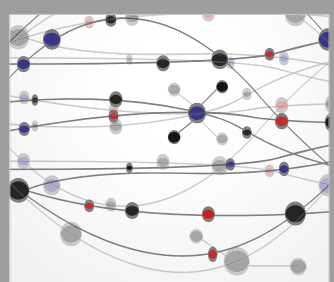

The Scientific World Journal
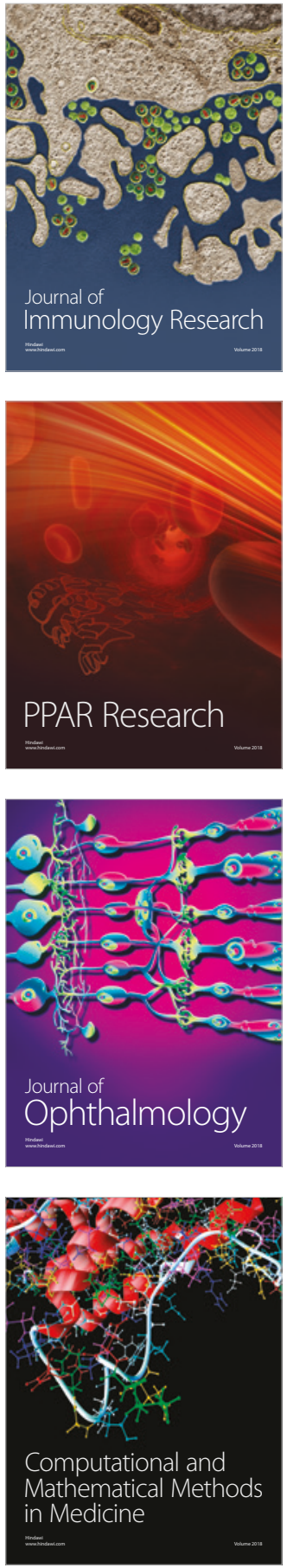

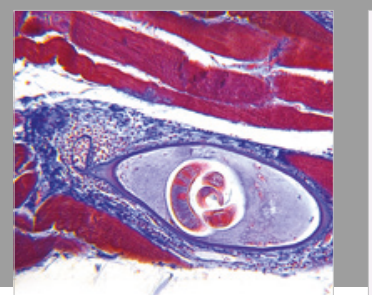

Gastroenterology Research and Practice

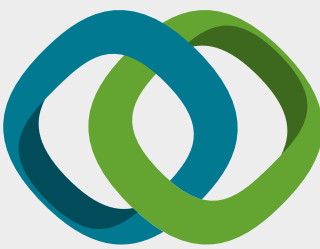

\section{Hindawi}

Submit your manuscripts at

www.hindawi.com
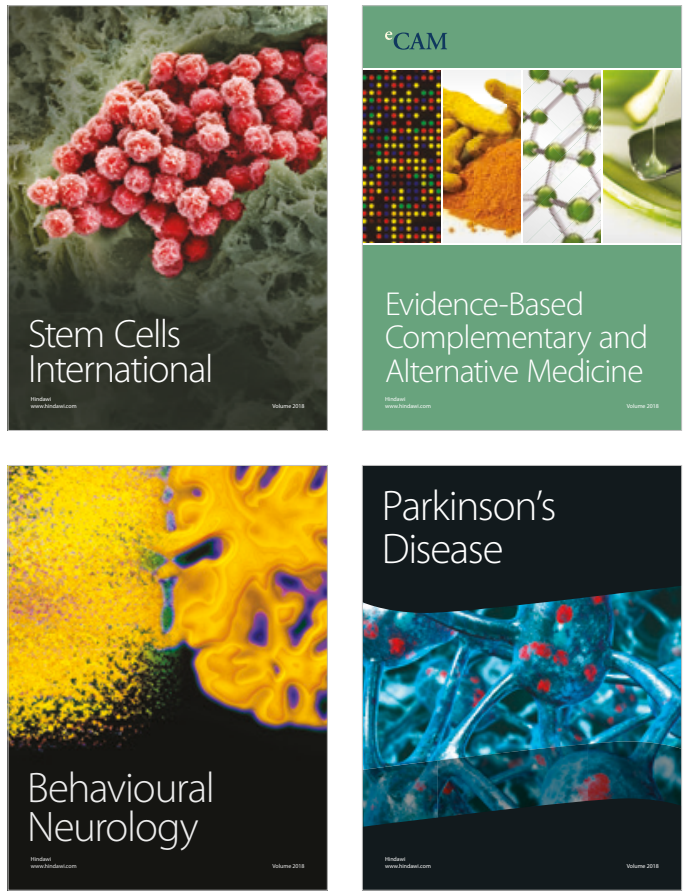

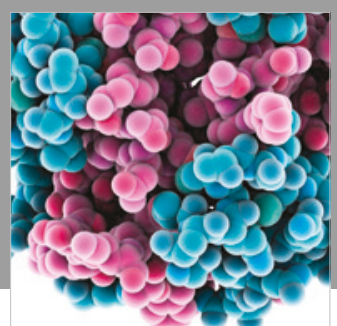

ournal of

Diabetes Research

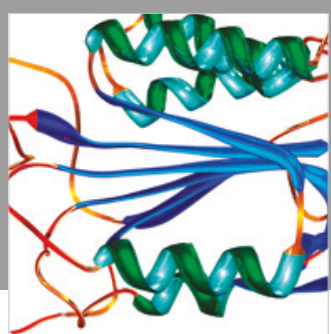

Disease Markers
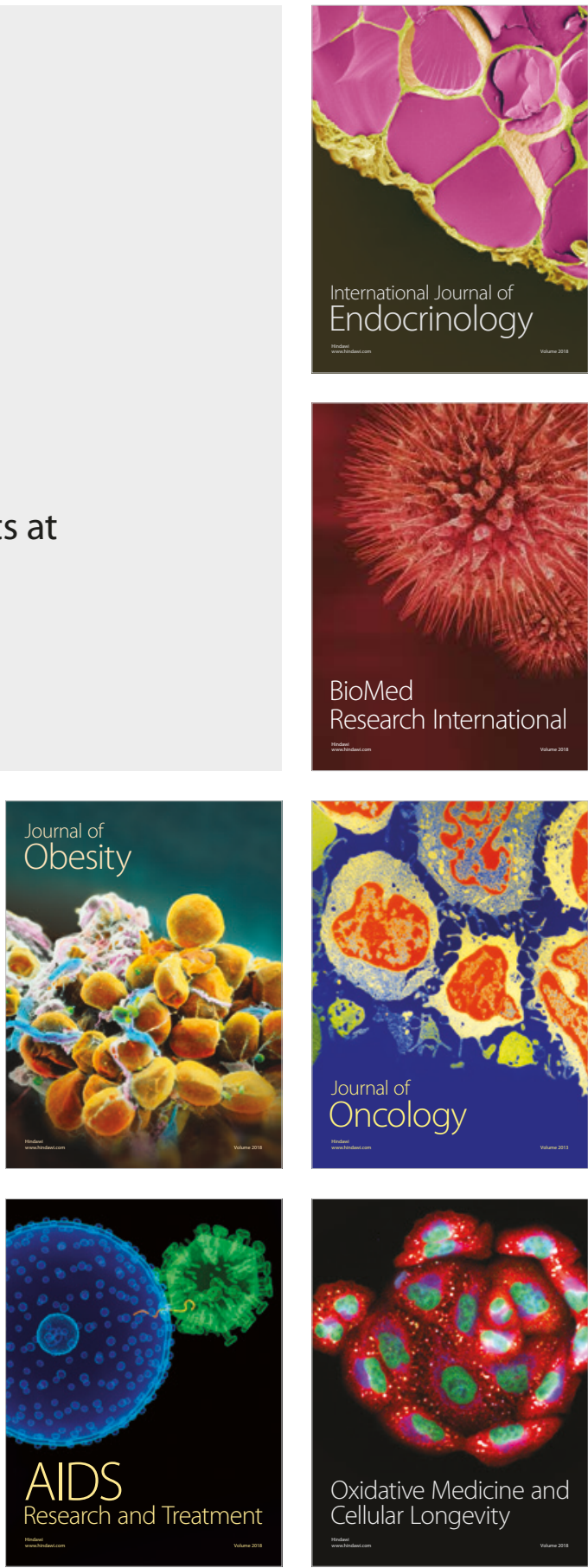